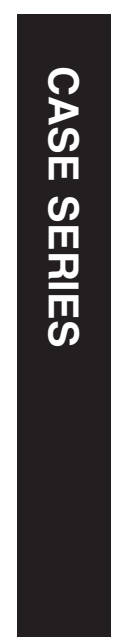

${ }^{1}$ Singapore Eye Research Institute, Singapore National Eye Centre, Singapore

${ }^{2}$ Department of Ophthalmology, National University of Singapore, Singapore

30uke-NUS Graduate Medical School, Singapore

Correspondence: SY Lee, Singapore National Eye Centre, 11 Third Hospital Avenue, Singapore 168751, Singapore. Tel: +65 62277255; Fax: +6562263395 E-mail: lee.shu.yen@ snec.com.sg

Received: 27 July 2013 Accepted in revised form: 25 November 2013

Published online:

17 January 2014

\title{
Comparison of spectral domain and swept-source optical coherence tomography in pathological myopia
}

\begin{abstract}
Purpose To compare optical coherence tomography (OCT) images obtained with swept-source OCT (SS-OCT) and spectral domain OCT (SD-OCT) in pathological myopia.
\end{abstract}

Methods This is a comparative observational cases series. Five patients with pathological myopia underwent SD-OCT and SS-OCT imaging. SS-OCT was performed using a prototype system (Topcon Medical Systems). SD-OCT was performed using enhanced depth imaging on the Heidelberg Spectralis OCT. The closest corresponding scans from the central subfield were compared.

Results Eight eyes of five patients with pathological myopia were included (mean spherical equivalent: $-16.00 \pm 4.70 \mathrm{D}$ ). Overall, SS-OCT better visualized retinochoroidal structures. The choroid, inner segment (IS)/outer segment (OS) line, and external limiting membrane (ELM) were clearly seen in a higher proportion of SS-OCT than SD-OCT scans, $(P<0.01$ for all $)$ whereas visualization of the sclera and retinal pigment epithelium (RPE) were similar. SS-OCT demonstrated foveoschisis in four eyes, with one of these not visible on SD-OCT. The wider SS-OCT scan revealed additional pathology not visible using SD-OCT along the staphyloma walls in $4 / 8$ images. These included incomplete posterior vitreous detachment in one eye and peripheral retinoschisis in $3 / 8$ eyes. Vitreoschisis was visible in $3 / 8$ SS-OCT images but not in the SD-OCT images. Conclusion SS-OCT is useful for imaging the posterior staphyloma of pathological myopia, providing greater detail than SD-OCT.
Eye (2014) 28, 488-491; doi:10.1038/eye.2013.308; published online 17 January 2014

Keywords: optical coherence tomography; pathological myopia; imaging; myopic foveoschisis

\section{Introduction}

Swept-source optical coherence tomography (SS-OCT) is a recent evolution of OCT employing a fast wavelength scanning light source. ${ }^{1}$ Compared with current spectral domain OCT (SD-OCT), SS-OCT has greater sensitivity and lower signal-to-noise ratios (SNR) at greater scanning depths.

OCT is invaluable in discerning subtle macular pathology in pathological myopia, ${ }^{2-5}$ but SD-OCT has significant limitations. The limited scanning depth frequently causes high SNRs, while the limited B-scan length does not image the entire width of the staphyloma and mirror artifacts are common. ${ }^{6}$

The aim of this paper is to compare OCT images obtained with SS-OCT and SD-OCT in pathological myopia.

\section{Materials and methods}

Five patients with pathological myopia underwent SD-OCT and SS-OCT scanning. SD-OCT was performed with the enhanced depth imaging mode of the Heidelberg Spectralis OCT system (Heidelberg Engineering, Vista, CA, USA). SS-OCT was performed using the Topcon Atlantis DRI OCT-1 system (Topcon Medical Systems, Paramus, NJ, USA). The system scan speed is $100000 \mathrm{~A}$-scans/s, 
using a light source with central wavelength of $1050 \mathrm{~nm}$ and bandwidth of $101 \mathrm{~nm}$. The axial resolution is $8.1 \mu \mathrm{m}$ and the degradation in sensitivity with depth is $2.4 \mathrm{~dB} / \mathrm{mm}$. $^{7}$ Scan lengths of $12 \mathrm{~mm}$ were used. All scans were performed by the same technician. Scans with motion artifacts were excluded, but poorer quality images due to media opacity were included to compare imaging capabilities in these situations. The closest corresponding scans from the same central subfield were selected for comparison.

\section{Results}

Eight eyes of five patients with posterior staphylomata were included (mean spherical equivalent refraction (SER): $-16.00 \pm 4.70 \mathrm{D}$; range: $-10.50 \mathrm{D}$ to $-23.00 \mathrm{D})$.

Overall, SS-OCT better visualized retino-choroidal structures (Table 1). The choroid, inner segment (IS)/ outer segment (OS) line, and external limiting membrane (ELM) were clearly seen in a higher proportion of SS-OCT than SD-OCT scans, $(P<0.01$ for all) while visualization of the sclera and retinal pigment epithelium (RPE) were similar.

The 12-mm SS-OCT scan imaged a wider extent of the staphyloma in $4 / 8$ eyes, whereas mirror artifacts were seen in the rest. The wider scans revealed additional pathology not visible using SD-OCT in 4/8 images. Insertion of the posterior hyaloid face with incomplete posterior vitreous detachment was seen in one case, and peripheral areas of retinoschisis in 3/8 eyes. Vitreoschisis with a persistent premacular vitreous attachment was visible in 3/8 SS-OCT images but not in SD-OCT images.

\section{Case reports}

\section{Case 1}

SS-OCT demonstrated myopic foveoschisis in four eyes. In one of these, the corresponding SD-OCT image did not demonstrate the foveoschisis clearly (Figure 1a and b) due to generally poor image quality.

\section{Case 2}

In this patient with SER of $-14.63 \mathrm{D}, \mathrm{SS}-\mathrm{OCT}$ of a peripheral area of inner retinoschisis clearly demonstrated bridging elements, distinguishing it from epiretinal tissue or internal limiting membrane (ILM) detachment (Figure 1c and d).

\section{Case 3}

Although SS-OCT evaluates a wider extent of the staphyloma, mirror artifacts are still present. In this patient (SER - 15.5D), additional areas of retinoschisis

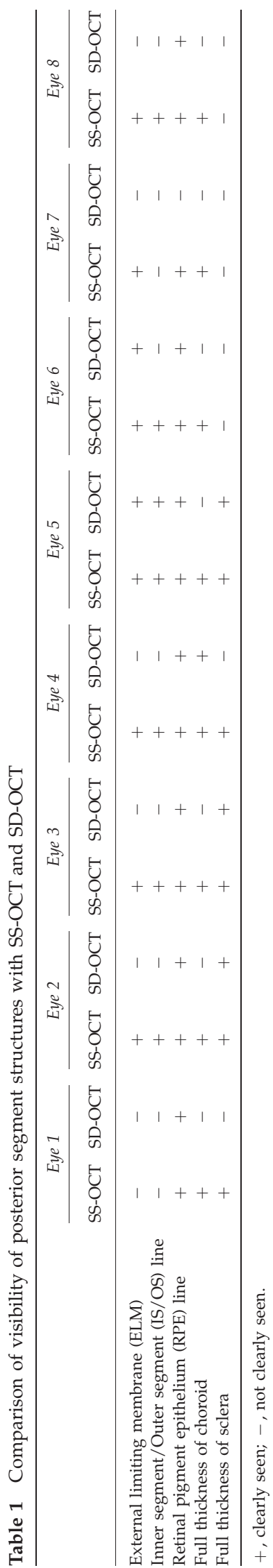




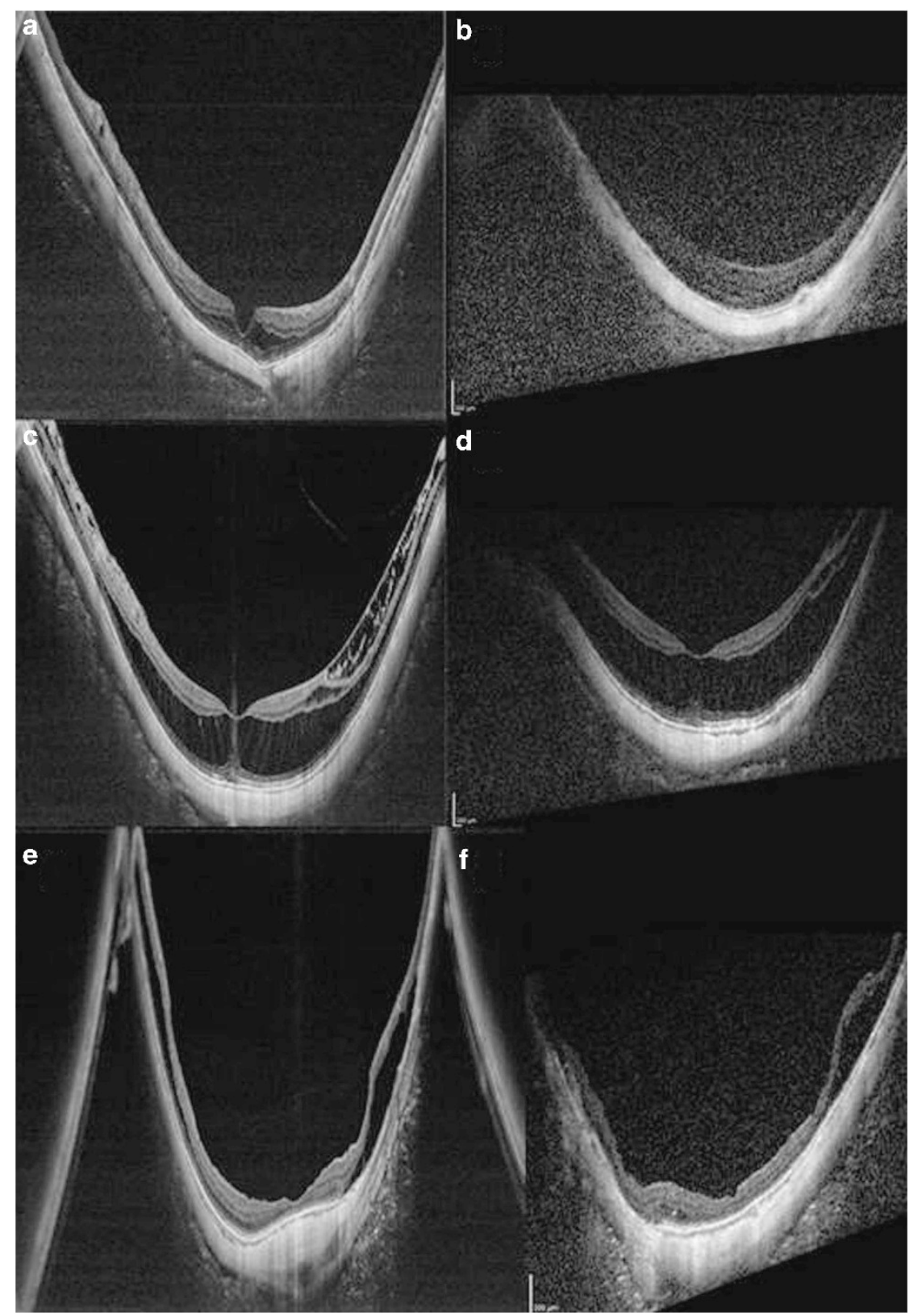

Figure 1 SS-OCT and corresponding SD-OCT patients of three cases. (a) SS-OCT demonstrated myopic foveoschisis clearly, but (b) SD-OCT does not, owing to generally poor image quality. (c) In this patient with a spherical equivalent refraction (SER) of $-14.63 \mathrm{D}$, SS-OCT of the peripheral area of inner retinoschisis clearly demonstrated bridging elements, distinguishing it from epiretinal tissue or internal limiting membrane (ILM) detachment. (d) The corresponding SD-OCT image does not show peripheral detail. (e) Wider extent of the staphyloma; mirror artifacts are still present. In this patient (SER - 15.5D), additional areas of retinoschisis are seen on the walls of the staphyloma on SS-OCT, and vitreoschisis is also visible. (f) SD-OCT does not image as wide an extent of the staphyloma and does not show as much detail.

are seen on the walls of the staphyloma on SS-OCT, and vitreoschisis is also visible (Figure 1e and $\mathrm{f}$ ).

\section{Discussion}

SS-OCT better images the posterior staphyloma in highly myopic eyes than SD-OCT, and a wider scan allows for the visualization of additional pathology.
SS-OCT better delineated the choroid but not the sclera than SD-OCT. In many cases, the extreme posterior protrusion of the staphyloma-limited visualization of the posterior scleral border. Morphological changes in the choroid and sclera are important as they have been implicated in the pathogenesis of myopia. ${ }^{5,7}$ The wider scan with SS-OCT detected pathology on the walls of the 
staphyloma and demonstrated the configuration of the staphyloma, but is still susceptible to mirror artifacts. $^{6}$

The extreme posterior protrusion and curvature of the posterior staphyloma of pathological myopia can adversely affect image resolution and SNRs. SS-OCT appears to allow better discrimination of retinal layers. The IS/OS line, the integrity of which has been correlated with visual acuity, ${ }^{8,9}$ was better seen with SS-OCT than SD-OCT. We were also able to demonstrate the anatomical changes of myopic foveoschisis more clearly with SS-OCT than SD-OCT, including distinct visualization of the bridging elements. The superior image quality with SS-OCT also allowed for visualization of the vitreous. Myopic traction maculopathy is frequently associated with a multi-laminar epiretinal complex composed of schitic vitreous, epiretinal membranes, and ILM. Surgical repair of myopic foveoschisis is more likely to be successful if all of these components are removed, and SS-OCT may aid in surgical planning. ${ }^{10,11}$ In some images, a clearer distinction between a detached ILM and a thin inner layer of a retinoschisis cavity could be made with SS-OCT (Figure 1c).

In conclusion, SS-OCT is a promising clinical and research tool in imaging the posterior staphyloma of pathological myopia. Further applications for the device in the near future are anticipated.

\section{Summary}

What was known before

- Optical coherence tomography is essential for evaluating macular pathology in high myopia.

- Spectral domain OCT performs poorly in imaging the posterior staphyloma of high myopia.

\section{What this study adds}

- New swept-source OCT technology provides superior imaging capabilities in pathological myopia.

\section{Conflict of interest}

The authors declare no conflict of interest.

\section{References}

1 Yasuno Y, Hong Y, Makita S, Yamanari M, Akiba M, Miura M et al. In vivo high-contrast imaging of deep posterior eye by 1-microm swept source optical coherence tomography and scattering optical coherence angiography. Opt Express 2007; 15: 6121-6139.

2 Panozzo G, Mercanti A. Optical coherence tomography findings in myopic traction maculopathy. Arch Opthalmol 2004; 122: 1455-1480.

3 Wang S, Peng Q, Zhao P. SD-OCT use in myopic retinoschisis pre- and post-vitrectomy. Optom Vis Sci 2012; 89: 678-683.

4 Baba T, Ohno-Matsui K, Futagami S, Yoshida T, Yasuzumi K, Kojima A. Prevalence and characteristics of foveal retinal detachment without macular hole in high myopi. Am J Opthalmol 2003; 135: 338-342.

5 Maruko I, Iida T, Sugano Y, Oyamada H, Akiba M, Sekiryu T. Morphologic analysis in pathologic myopia using high-penetration optical coherence tomography. Invest Ophthalmol Vis Sci 2012; 53: 3834-3838.

6 Ho J, Castro DP, Castro LC, Chen Y, Liu J, Mattox C et al. Clinical assessment of mirror artifacts in spectral-domain optical coherence tomography. Invest Ophthalmol Vis Sci 2010; 51: 3714-3720.

7 Spaide RF, Akiba M, Ohno-Matsui K. Evaluation of peripapillary intrachoroidal cavitation with swept source and enhanced depth imaging optical coherence tomography. Retina 2012; 32: 1037-1044.

8 Itoh Y, Inoue M, Rii T, Hiraoka T, Hirakata A. Correlation between length of foveal cone outer segment tips line defect and visual acuity after macular hole closure. Ophthalmology 2012; 119: 1438-1446.

9 Chalam KV, Murthy RK, Gupta SK, Brar VS, Grover S. Foveal structure defined by spectral domain optical coherence tomography correlates with visual function after macular hole surgery. Eur J Ophthalmol 2010; 20: 572-577.

10 Ho TC, Chen MS, Huang JS, Shih YF, Ho H, Huang YH. Foveola nonpeeling technique in internal limiting membrane peeling of myopic foveoschisis surgery. Retina 2012; 32: 631-634.

11 Kim KS, Lee SB, Lee WK. Vitrectomy and internal limiting membrane peeling with and without gas tamponade for myopic foveoschisis. Am J Ophthalmol 2012; 153: 320-326. 